action, increased before lever-pressing in mice that were willing to obtain shocks along with dopamine self-stimulation. Optogenetic inhibition of this neural pathway turned persevering mice into renouncing mice. This finding shows that the increased activity of neurons projecting from the OFC to the dorsal striatum was necessary for this form of compulsive activation of dopamine neurons.

However, this behavioural switch was only temporary: when optogenetic inhibition was turned off, the compulsive behaviour resumed in persevering mice. The authors reasoned that long-lasting changes at the synapses - the junctions between neurons - that connect OFC and dorsal striatum neurons could arise as a result of the many days of self-stimulation of dopamine neurons. If these changes occurred only in persevering mice, this would explain their persistent compulsive behaviour.

If this hypothesis is true, the strength of synaptic connections between OFC and dorsal striatum neurons should be greater in perseverers than in renouncers, enabling better activation of dorsal striatum neurons by OFC neurons. Indeed, Pascoli et al. went on to show that the strength of the synapses between OFC neurons and dorsal striatum neurons had increased in persevering mice (Fig. 1). Renouncers, along with mice that had never been exposed to the experimental setup and mice that received shocks but were not allowed to use the lever, all showed low synaptic strength between OFC and dorsal striatum neurons.

Remarkably, the authors found that compulsive behaviour could be suppressed or induced by respectively decreasing or increasing the strength of this neural connection. Weakening of the synaptic connections between the OFC and the dorsal striatum in persevering mice reduced their willingness to self-stimulate in the face of a possible foot shock. Conversely, renouncers could be turned into perseverers by increasing the strength of these synaptic connections. In contrast to the temporary reversal observed after optogenetic inhibition of OFC neurons projecting to the dorsal striatum, these changes in synaptic strength induced a behavioural switch that persisted for six days.

Pascoli et al. have discovered a neuroadaptation that allows mice to override a painful stimulus to continue activating their dopamine neurons. The chronic consumption of drugs of abuse in humans leads to repeated activation of the same dopamine-reinforcement circuit, so a similar neuroadaptation might cause them to continue taking drugs despite the negative consequences. To test this proposition, we should determine whether changes in the strength of the connections between OFC and dorsal striatum neurons mediate compulsive behaviour in mice pressing a lever to receive cocaine, amphetamines or opioids in the face of a possible foot shock.

Does the optogenetic stimulation of dopamine neurons accurately mimic the activation of dopamine neurons by drugs of abuse? There are obvious differences between quickly switching a laser on and off during optogenetic stimulation and the slower onset and longer duration of drug action. Nevertheless, the authors previously showed ${ }^{4}$ that cocaine intake and optogenetic activation induce almost identical adaptations in dopamine neurons and their immediate downstream targets, providing a strong rationale for the experimental approach used in the current study.

Why does the self-stimulation of dopamine neurons lead to compulsive behaviour in only a subset of individuals? Persevering and renouncing mice self-stimulated for approximately the same time and with a similar number of events before foot-shock punishments began, yet the brains of the two groups seem to have changed in divergent ways. The VTA dopamine neurons stimulated by the mice do not connect directly to the OFC or the dorsal striatum, so the link between these regions must involve multiple synaptic connections. A multisynaptic route through which the activation of VTA dopamine neurons might cause changes in the dorsal striatum has previously been described ${ }^{5}$, and has been proposed to underlie transitions from noncompulsive to compulsive drug-taking ${ }^{6,7}$. Pre-existing differences in this multisynaptic circuit might explain why compulsive behaviour, and the related changes in synaptic connections, occur in only some mice.

Synaptic changes can last for days, years or even a lifetime. Might the changes discovered by Pascoli et al. form the basis of an enduring behavioural change that is a hallmark of drug addiction? Resolving this question will require experimental evidence that drug self-administration despite negative consequences occurs through strengthening of the connections between the OFC and the dorsal striatum, and that it is indeed the activation of dopamine systems that sets in motion a chain of neural events that culminates in compulsive drug-taking.

Patricia Janak is in the Departments of Neuroscience and Psychological \& Brain Sciences, Johns Hopkins University, Baltimore, Maryland 21218, USA.

e-mail:patricia.janak@jhu.edu

1. Pascoli, V. et al. Nature 564, 366-371 (2018).

2. American Psychiatric Association. Diagnostic and Statistical Manual of Mental Disorders 5th edn (Am. Psychiatr. Assoc., 2013).

3. Pascoli, P., Terrier, J., Hiver, A. \& Lüscher, C. Neuron 88, 1054-1066 (2015)

4. Brown, M. T. C., Korn, C. \& Lüscher, C. Channels 5, 461-463 (2011)

5. Haber, S. N., Fudge, J. L. \& McFarland, N. R. J. Neurosci. 20, 2369-2382 (2000).

6. Everitt, B. J. \& Robbins, T. W. Annu. Rev. Psychol. 67 23-50 (2016).

7. Keiflin, R. \& Janak, P. H. Neuron $88,247-263$ (2015).

\title{
Elusive torque sensed by liquid crystals
}

\begin{abstract}
Almost half a century ago, it was predicted that the confinement of quantum fluctuations could induce mechanical rotation - the Casimir torque. This prediction has now been confirmed using liquid crystals. SEE LETTER P.386
\end{abstract}

\section{SLOBODAN ŽUMER}

Q uantum physics tells us that empty space is filled with fluctuating electromagnetic fields. If two metal plates are positioned close to each other, the quantum fluctuations between the plates differ from those outside the plates, producing a force that pushes the plates closer together. This phenomenon is known as the Casimir effect. In 1972, it was suggested ${ }^{1}$ that quantum fluctuations could also generate a turning effect, called a torque, if the metal plates were replaced by materials that are optically anisotropic - that is, their optical properties, sensed by a light beam, depend on the beam's direction. On page 386, Somers et al. ${ }^{2}$ report experimental evidence for this Casimir torque through the twisting of liquid crystals. The discovery paves the way for the development of complex micrometre- and nanometre-scale mechanical devices.

Following the prediction of the Casimir effect between two ideal metal plates ${ }^{3}$, the concept was extended to real materials, such as conventional metals and electrical insulators known as dielectrics ${ }^{4}$. The Casimir effect can be explained by a restriction in the quantum and thermal fluctuations that can exist between the boundaries of two materials, leading to a weak attractive force. This force is maximal if the confining boundaries are identical, and is smaller - or can even be repulsive - if the boundaries differ in their electrical properties or shape ${ }^{5}$.

Because of the weakness of the Casimir force and its strong dependence on confining boundaries, it was nearly 50 years before solid experimental confirmation of the Casimir 


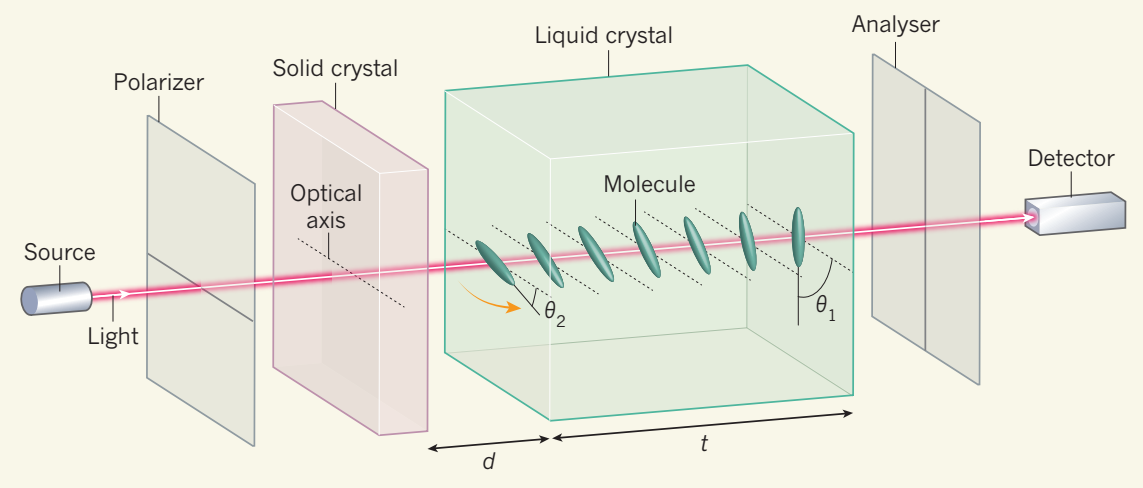

Figure 1 | Measurement of the Casimir torque. Somers et al. ${ }^{2}$ have demonstrated that a solid crystal can cause the average orientation of molecules in a 'nematic' liquid crystal to twist (from angle $\theta_{1}$ to angle $\theta_{2}$ ), owing to a phenomenon known as the Casimir torque (orange arrow). The authors detected light that passed from a source through a polarizer, the two crystals and a second polarizer called an analyser. The thickness $(t)$ of the liquid crystal was much larger than both the distance $(d)$ between the crystals and the wavelength of the light. The authors selected a particular value for $\theta_{1}$ and the orientations of the polarizers, and varied the solid crystal's optical axis - a direction in the crystal along which the speed of propagating light is independent of the light's polarization. They determined $\theta_{2}$ from the intensity of the transmitted light, and measured the Casimir torque using the value of $\theta_{2}$ and the known elastic properties of the liquid crystal.

effect was realized ${ }^{6}$. Concurrently, it became clear that the concept of fluctuation-induced interactions, arising from confined fluctuating electromagnetic fields, could be generalized to other confined fields in different areas of physics. Examples of such fields include sound waves and capillary waves (ripples).

Other fluidic systems that have fluctuation-induced interactions include 'critical' fluids ${ }^{7}$ and liquid crystals ${ }^{8}$. In the latter, the molecular ordering associated with different crystal phases - such as cholesteric, nematic and smectic phases - and, in particular, the anchoring of liquid crystals to surfaces can lead to diverse behaviour of Casimir-like forces ${ }^{9}$. It is also worth mentioning examples beyond condensed-matter systems, such as confined gravitational waves ${ }^{10}$.

The realization that altering the properties of the confining boundaries changes the ensuing Casimir force was naturally followed by the idea that breaking the symmetry of physical properties of confining surfaces or objects could lead to the emergence of the Casimir torque $e^{5}$. More than four decades ago, a theory was developed ${ }^{1}$ to describe the Casimir torque between two solid, optically anisotropic crystals that are in close proximity to each other. This theory was subsequently improved and extended to morecomplex systems such as layered media ${ }^{11}$, and many experiments were proposed. Nevertheless, it is only now, with the work of Somers and colleagues, that the existence of the Casimir torque has been convincingly proved.

The authors' groundbreaking experiment was based on the idea ${ }^{12}$ of replacing one of the two solid crystals with a nematic liquid crystal. The liquid crystal was fixed on one side, and was exposed to the nearby solid crystal, which was free to rotate, on the other side (Fig. 1).

The liquid crystal had two roles: first as an optically anisotropic material, and second as a torque sensor.

The Casimir torque, although weak, forced the average orientation of molecules in the nematic liquid crystal along a direction that characterizes the solid crystal, known as its optical axis. This produced a twisted deformation that spread through the whole of the liquid crystal. Somers et al. detected the deformation by its effect on the intensity of light that was passed through a polarizer, the two crystals and a second polarizer called the analyser (Fig. 1). This clever experimental

\section{"The authors'} observation is a key contribution to fundamental physics that also has broad implications." set-up allowed the authors to determine the dependence of the Casimir torque on the distance between the crystals, for four different solid crystals.

Liquid crystals offer great potential because of their large response to weak external electric fields and other perturbations. Their best-known application is in liquid-crystal displays (LCDs). A less-known application is their use as sensor elements. Temperature sensors based on the temperature sensitivity of light reflection from cholesteric liquid crystals have been used for decades. By contrast, chemical sensors based on the adsorption of molecules on liquid-crystal surfaces, particularly for sensing biological molecules, have been developed only in the past decade. Somers and colleagues' sensor for the Casimir torque is the latest example of a sensor in which a minuscule torque is determined using the elasticity of a liquid crystal, rather than by conventional mechanical means.
One remaining question is whether the measured Casimir torque could be enhanced. Much progress has been made in the optimization of nematic liquid crystals for displays and photonic applications, and so it would be worth exploring whether a liquid crystal is available that has more-optimal properties than has the crystal used by the authors. In addition to increasing the torque, such a liquid crystal would need to be more easily twisted than the one used here.

It is also possible that the torque sensor could be made from a cholesteric liquid crystal. Such a crystal selectively reflects circularly polarized light that has a polarization rotating in the same direction as the crystal's intrinsically twisted structure. The Casimir torque would further twist, or untwist, the cholesteric crystal's structure, affecting the selective light reflection in a way that should be detectable.

The successful observation of the Casimir torque by Somers et al. is a key contribution to fundamental physics that also has broad implications. The evolution of microscale mechanical and electromechanical devices has already reached to below the micrometre scale, and needs to take into account quantum phenomena and the effects of thermal and quantum fluctuations. Therefore, the development of nanoscale mechanical and electromechanical systems must take into account the Casimir force and torque, or even directly use these phenomena ${ }^{13}$.

Building on the current work, Casimir-like effects that can occur because of thermal fluctuations should be examined in many confined systems, including gases, conventional liquids, critical liquids, colloidal dispersions, polymers and liquid crystals. This could enable these systems to be used in complex micro- and nanoscale fluidic devices.

Slobodan Žumer is in the Department of Physics, Faculty of Mathematics and Physics, University of Ljubljana, and the Jožef Stefan Institute, 1000 Ljubljana, Slovenia.

e-mail:slobodan.zumer@fmf.uni-lj.si

1. Parsegian, V. A. \& Weiss, G. H. J. Adhes. 3, 259-267 (1972).

2. Somers, D. A. T., Garrett, J. L., Palm, K. J. \& Munday, J. N. Nature 564, 386-389 (2018)

3. Casimir, H. B. G. Proc. K. Ned. Akad. Wet. 51, 793-795 (1948).

4. Lifshitz, E. M. J. Exp. Theor. Phys. USSR 29, 94-110 (in Russian) (1955); Sov. Phys. 2, 73-83 (1956).

5. Woods, L. M. et al. Rev. Mod. Phys. 88, 045003 (2016)

6. Lamoreaux, S. K. Phys. Rev. Lett. 78, 5-8 (1997)

7. Hertlein, C., Helden, L., Gambassi, A., Dietrich, S. \& Bechinger, C. Nature 451, 172-175 (2008).

8. Ajdari, A., Peliti, L. \& Prost, J. Phys. Rev. Lett. 66, 1481-1484 (1991).

9. Ziherl, P., Podgornik, R. \& Žumer, S. Chem. Phys. Lett. 95, 99-104 (1998).

10.Quach, J. Q. Phys. Rev. Lett. 114, 081104 (2015).

11.Lu, B.-S. \& Podgornik, R. J. Chem. Phys. 145, 044707 (2016).

12.Smith, E. R. \& Ninham, B. W. Physica 66, 111-130 (1973).

13. Capasso, F., Munday, J. N., lannuzzi, D. \& Chan, H. B. IEEE J. Sel. Top. Quantum Electron. 13, 400-414 (2007). 